\title{
CUBIC SYMMETROIDS AND VECTOR BUNDLES ON A QUADRIC SURFACE
}

\author{
SUKMOON HUH
}

\begin{abstract}
We investigate the jumping conics of stable vector bundles $\mathcal{E}$ of rank 2 on a smooth quadric surface $Q$ with the Chern classes $c_{1}=$ $\mathcal{O}_{Q}(-1,-1)$ and $c_{2}=4$ with respect to the ample line bundle $\mathcal{O}_{Q}(1,1)$. We describe the set of jumping conics of $\mathcal{E}$, a cubic symmetroid in $\mathbb{P}_{3}$, in terms of the cohomological properties of $\mathcal{E}$. As a consequence, we prove that the set of jumping conics, $S(\mathcal{E})$, uniquely determines $\mathcal{E}$. Moreover we prove that the moduli space of such vector bundles is rational.
\end{abstract}

\section{IntRoduction}

Throughout the article, our base field is $\mathbb{C}$, the complex numbers.

Let $Q$ be a smooth quadric in $\mathbb{P}_{3}=\mathbb{P}(V)$, where $V$ is a 4-dimensional vector space over complex numbers $\mathbb{C}$, and $\mathfrak{M}(k)$ be the moduli space of stable vector bundles of rank 2 on $Q$ with the Chern classes $c_{1}=\mathcal{O}_{Q}(-1,-1)$ and $c_{2}=k$ with respect to the ample line bundle $\mathcal{L}=\mathcal{O}_{Q}(1,1) . \mathfrak{M}(k)$ forms an open Zariski subset of the projective variety $\overline{\mathfrak{M}}(k)$ whose points correspond to the semi-stable sheaves on $Q$ with the same numerical invariants. The Zariski tangent space of $\mathfrak{M}(k)$ at $\mathcal{E}$, is naturally isomorphic to $H^{1}(Q, \mathcal{E} n d(\mathcal{E}))$ [7] and so the dimension of $\mathfrak{M}(k)$ is equal to $h^{1}(Q, \mathcal{E} n d(\mathcal{E}))=4 k-5$, since $\mathcal{E}$ is simple.

In [5], we define the jumping conics of $\mathcal{E} \in \mathfrak{M}(k)$ as a point in $\mathbb{P}_{3}^{*}$ and prove that the set of jumping conic is a symmetric determinantal hypersurface of degree $k-1$ in $\mathbb{P}_{3}^{*}$. It enables us to consider a morphism

$$
S: \mathfrak{M}(k) \rightarrow\left|\mathcal{O}_{\mathbb{P}_{3}^{*}}(k-1)\right| \simeq \mathbb{P}_{N} .
$$

The conjecture is that the general $\mathcal{E} \in \mathfrak{M}(k)$ is uniquely determined by $S(\mathcal{E})$. It is true in the case of $k \leq 3[5]$.

In this article, we prove that the conjecture is true in the case of $k=4$. For a stable vector bundle $\mathcal{E}$ in $\mathfrak{M}(4), S(\mathcal{E})$ is a cubic symmetroid surface, i.e. a symmetric determinantal cubic hypersurface in $\mathbb{P}_{3}^{*}$. In terms of short exact sequences that $\mathcal{E}$ admits, we can obtain the relation between the singularity of $S(\mathcal{E})$ and the dimension of cohomology of the restriction of $\mathcal{E}$ to

1991 Mathematics Subject Classification. Primary: 14D20; Secondary: 14E05.

Key words and phrases. Jumping conics, stable bundle, quadric surface, determinantal variety.

The author is supported by Basic Science Research Program 2012-0002904 through NRF funded by MEST. 
its hyperplane section. It turns out that $S(\mathcal{E})$ has exactly 4 singular points. It eventually enables us to prove the rationality of $\mathfrak{M}(4)$.

In the last part of the article, we give a brief description of $S(\mathcal{E})$ for non-general vector bundles of $\mathfrak{M}(4)$.

We will denote the dimension of the cohomology $H^{i}(X, \mathcal{F})$ for a coherent sheaf $\mathcal{F}$ on $X$ by $h^{i}(X, \mathcal{F})$, or simply by $h^{i}(\mathcal{F})$ if there is no confusion.

\section{Preliminaries}

Let $Q$ be a smooth quadric surface isomorphic to $\mathbb{P}\left(V_{1}\right) \times \mathbb{P}\left(V_{2}\right)$ for two 2-dimensional vector spaces $V_{1}$ and $V_{2}$. Then it is embedded into $\mathbb{P}_{3} \simeq \mathbb{P}(V)$ by the Segre map, where $V=V_{1} \otimes V_{2}$. Let us denote $f^{*} \mathcal{O}_{\mathbb{P}_{1}}(a) \otimes g^{*} \mathcal{O}_{\mathbb{P}_{1}}(b)$ by $\mathcal{O}_{Q}(a, b)$ and $\mathcal{E} \otimes \mathcal{O}_{Q}(a, b)$ by $\mathcal{E}(a, b)$ for coherent sheaves $\mathcal{E}$ on $Q$, where $f$ and $g$ are the projections from $Q$ to each factors. Then the canonical line bundle $K_{Q}$ of $Q$ is $\mathcal{O}_{Q}(-2,-2)$.

Lemma 2.1. We have

$$
H^{i}\left(Q, \mathcal{O}_{Q}(a, a+b)\right)= \begin{cases}0, & \text { if } a=-1 \\ H^{i}\left(\mathbb{P}_{1}, \mathcal{O}_{\mathbb{P}_{1}}(a+b)^{\oplus(a+1)}\right), & \text { if } a \geq 0\end{cases}
$$

The other cases can be induced from the above by the Serre duality and symmetry.

Proof. Note that $Q$ is the projectivization of the vector bundle $\mathcal{O}_{\mathbb{P}_{1}}(1)^{\oplus 2}$ over $\mathbb{P}_{1}$. Then the proof follows from the degeneration of the Leray spectral sequence

$$
H^{i}\left(\mathbb{P}_{1}, R^{j} f_{*} \mathcal{O}_{Q}(a, a+b)\right) \Rightarrow H^{i+j}\left(Q, \mathcal{O}_{Q}(a, a+b)\right)
$$

Now let us denote the ample line bundle $\mathcal{O}_{Q}(1,1)$ by $\mathcal{L}$.

Definition 2.2. A torsion free sheaf $\mathcal{E}$ of rank $r$ on $Q$ is called stable (resp. semi-stable) with respect to $\mathcal{L}$ if

$$
\frac{\chi\left(\mathcal{F} \otimes \mathcal{L}^{\otimes m}\right)}{r^{\prime}}<(\text { resp. } \leq) \frac{\chi\left(\mathcal{E} \otimes \mathcal{L}^{\otimes m}\right)}{r},
$$

for all non-zero subsheaves $\mathcal{F} \subset \mathcal{E}$ of rank $r^{\prime}$.

Let $\overline{\mathfrak{M}}(k)$ be the moduli space of semi-stable sheaves of rank 2 on $Q$ with the Chern classes $c_{1}=\mathcal{O}_{Q}(-1,-1)$ and $c_{2}=k$ with respect to $\mathcal{L}=\mathcal{O}_{Q}(1,1)$. The existence and the projectivity of $\overline{\mathfrak{M}}(k)$ is known in [3] and it has an open Zariski subset $\mathfrak{M}(k)$ which consists of the stable vector bundles with the given numeric invariants. By the Bogomolov theorem [7, $\mathfrak{M}(k)$ is empty if $4 k<c_{1}^{2}=2$ and in particular, we can consider only the case of $k \geq 1$. The dimension of $\mathfrak{M}(k)$ can be computed to be $h^{1}(Q, \mathcal{E} n d(\mathcal{E}))=4 k-5$.

Note that $\mathcal{E} \simeq \mathcal{E}^{*}(-1,-1)$ and by the Riemann-Roch theorem [4], we have

$$
\chi_{\mathcal{E}}(m):=\chi(\mathcal{E}(m, m))=2 m^{2}+2 m+1-k,
$$

for $\mathcal{E} \in \overline{\mathfrak{M}}(k)$. 
For a hyperplane section $H$ of $\mathbb{P}_{3}$, we define $C_{H}:=Q \cap H$ to be a conic on $H$.

Definition 2.3. The conic $C_{H}$ is called to be a jumping conic if $h^{0}\left(\left.\mathcal{E}\right|_{C_{H}}\right) \geq$ 1 and let

$$
S(\mathcal{E}):=\left\{H \in \mathbb{P}_{3}^{*} \mid h^{0}\left(\left.\mathcal{E}\right|_{C_{H}}\right) \geq 1\right\} .
$$

When $C_{H}$ is a smooth conic on $H$, it is a jumping conic if the vector bundle $\mathcal{E}$ splits non-generically over it.

Theorem 2.4. [5] For $\mathcal{E} \in \mathfrak{M}(k), S(\mathcal{E})$ is a symmetric determinantal hypersurface of degree $k-1$ in $\mathbb{P}_{3}^{*}$ and it has a singular point at $H \in \mathbb{P}_{3}^{*}$ if and only if $h^{0}\left(\left.\mathcal{E}\right|_{C_{H}}\right) \geq 2$.

It enables us to consider a morphism

$$
S: \mathfrak{M}(k) \rightarrow\left|\mathcal{O}_{\mathbb{P}_{3}^{*}}(k-1)\right| \simeq \mathbb{P}_{N},
$$

where $N=\left(\begin{array}{c}k+2 \\ 3\end{array}\right)-1$.

In [5] 6], the cases of $k=2,3$ are dealt in detail. For example, when $k=2$, the morphism $S$ extends to an isomorphism from $\overline{\mathfrak{M}}(2) \rightarrow \mathbb{P}_{3}$ and $\mathfrak{M}(2)$ is isomorphic to $\mathbb{P}_{3} \backslash Q$. In particular, $S(\mathcal{E})$ determines uniquely $\mathcal{E} \in \mathfrak{M}(2)$. An analogue of this result turns out to be true in the case of $k=3$.

\section{Results}

From now on, we will investigate $S(\mathcal{E})$ for $\mathcal{E} \in \mathfrak{M}(4)$, which is now a cubic symmetroid surface, i.e. a symmetric determinantal cubic surface in $\mathbb{P}_{3}^{*}$. Note that a nonsingular cubic surface cannot be symmetrically determinantal [2].

Since $\chi_{\mathcal{E}}(1)=1$ and $\mathcal{E}$ is stable, it admits an exact sequence

$$
0 \rightarrow \mathcal{O}_{Q} \rightarrow \mathcal{E}(1,1) \rightarrow \mathcal{I}_{Z}(1,1) \rightarrow 0,
$$

where $Z$ is a zero-dimensional subscheme of $Q$ with length 4 and $\mathcal{I}_{Z}(1,1)$ is the tensor product of the ideal sheaf of $Z$ and $\mathcal{O}_{Q}(1,1)$. Let us assume that $Z$ is in general position, then we can check that $h^{0}(\mathcal{E}(1,1))=1$. In particular, $Z$ is uniquely determined by $\mathcal{E}$. Note that

$$
\mathbb{P} \operatorname{Ext}^{1}\left(\mathcal{I}_{Z}(1,1), \mathcal{O}_{Q}\right) \simeq \mathbb{P} H^{0}\left(\mathcal{O}_{Z}\right)^{*} \simeq \mathbb{P}_{3} .
$$

A general point in this family of extensions corresponds to a stable vector bundle [1] and so $\mathfrak{M}(4)$ is birational to a $\mathbb{P}_{3}$-bundle over the Hilbert scheme $Q^{[4]}$ of zero-dimensional subscheme of $Q$ with length 4 . It is consistent with the fact that the dimension of $\mathfrak{M}(4)$ is 11 . Note that $Q^{[4]}$ is a resolution of singularity of $S^{4} Q$, the 4 th symmetric power of $Q$, and in particular it is 8-dimensional 8 .

Assume that $Z$ is not contained in any hyperplane section. If $|Z \cap H|=3$, for a hyperplane section $H$ of $\mathbb{P}_{3}^{*}$, we obtain the following exact sequence by tensoring the sequence (11) with $\mathcal{O}_{C_{H}}$ :

$$
\left.0 \rightarrow \mathcal{O}_{C_{H}}(p) \rightarrow \mathcal{E}\right|_{C_{H}} \rightarrow \mathcal{O}_{C_{H}}(-3 p) \rightarrow 0,
$$


where $p$ is a point on $C_{H}$. Since $h^{0}\left(\left.\mathcal{E}\right|_{C_{H}}\right)=2, H$ is a singular point of $S(\mathcal{E})$.

Similarly we can prove that $H$ is a non-singular point of $S(\mathcal{E})$ if $|Z \cap H|=$ 2, and not a point of $S(\mathcal{E})$ if $|Z \cap H|=1$. If $H$ does not contain any point of $Z$, then we have the sequence

$$
\left.0 \rightarrow \mathcal{O}_{C_{H}}(-2 p) \rightarrow \mathcal{E}\right|_{C_{H}} \rightarrow \mathcal{O}_{C_{H}} \rightarrow 0
$$

and the dimension of $\operatorname{Ext}^{1}\left(\mathcal{O}_{C_{H}}, \mathcal{O}_{C_{H}}(-2 p)\right)$ is 1 . So it is not obvious whether $H$ is a point of $S(\mathcal{E})$ or not. But if it is a point of $S(\mathcal{E})$, it would not be a singular point. So there are exact 4 singular points of $S(\mathcal{E})$.

Let us fix a line $l$ in $\mathbb{P}_{3}$. Let us consider the $\mathbb{P}_{1}$-family of hyperplanes of $\mathbb{P}_{3}$ that contains $l$, say $\left\{H_{s}\right\}, s \in \mathbb{P}_{1}$. If $l$ passes through two points of $Z$, then there are two hyperplanes in the family $\left\{H_{s}\right\}$ that contain three points of $Z$. In other words, the projective line in $\mathbb{P}_{3}^{*}$ corresponding to the family intersects with $S(\mathcal{E})$ at two singular points. Since $S(\mathcal{E})$ is a cubic hypersurface, so the projective line must be contained in $S(\mathcal{E})$. Summarizing the argument so far, we obtain:

Proposition 3.1. For a general vector bundle $\mathcal{E}$ in $\mathfrak{M}(4)$, there are exactly 4 singular points of $S(\mathcal{E})$ and exactly 6 lines contained in $S(\mathcal{E})$.

Proof. The 6 lines connecting two of the 4 singular points are contained in $S(\mathcal{E})$ and so it is enough to check that they are all. Let $Z=\left\{p_{1}, \cdots, p_{4}\right\}$ and denote the line connecting $p_{i}$ and $p_{j}$ by $l_{i j}$. Let us assume that there exists a line $l$ which is different from $l_{i j}$. As the first case, let us assume that $l$ does not intersect with $l_{i j}$. If $\pi: \mathbb{P}_{3}^{*} \rightarrow \mathbb{P}_{2}^{*}$ is the projection from $p_{1}$, then the images of $l$ and $l_{i j}, i, j \neq 1$ intersect. It implies that $l$ and $l_{i j}$ intersect for $i, j \neq 2$. But this is impossible since the plane containing $p_{2}, p_{3}, p_{4}$ would contain $l$ and so .

The case when $l$ intersects with $l_{i}$ can be shown to be impossible in a similar way.

Remark 3.2. When we consider a $\mathbb{P}_{2}$-family of hyperplanes of $\mathbb{P}_{3}$ that contains a point of $Z$, the intersection of $\mathbb{P}_{2}$ with $S(\mathcal{E})$ is a cubic plane curve. And in fact, there are 3 hyperplanes in this family, that contain 3 points of $Z$. Thus the intersection of the $\mathbb{P}_{2}$-family with $S(\mathcal{E})$ is the union of three lines.

Conversely, let us consider a cubic hypersurface $S_{3}$ in $\mathbb{P}_{3}^{*}$ with 4 singular points, say $H_{1}, \cdots, H_{4} \subset \mathbb{P}_{3}$. Then $H_{i}$ 's are 4 hyperplanes of $\mathbb{P}_{3}$ in general position. If $S_{3}$ is equal to $S(\mathcal{E})$ for some $\mathcal{E} \in \mathfrak{M}(4)$ with the exact sequence (11), then there are 3 points of $Z$ on each $H_{i}$. The intersection of $C_{H_{1}}$ with $H_{i}, i=2,3,4$ is two points of $Z$ and so 3 points of $Z$ are determined. The last point is just the intersection of $\mathrm{H}_{2}, \mathrm{H}_{3}$ and $\mathrm{H}_{4}$.

Theorem 3.3. The morphism $S: \mathfrak{M}(4) \rightarrow\left|\mathcal{O}_{\mathbb{P}_{3}^{*}}(3)\right|$ is generically oneto-one. In other words, the set of jumping conics of $\mathcal{E} \in \mathfrak{M}(4)$, uniquely determines $\mathcal{E}$ in general. 
Proof. It is enough to check that for two different stable vector bundles $\mathcal{E}$ and $\mathcal{E}^{\prime}$ that fit into the sequence (1) with the same $Z, S(\mathcal{E})$ and $S\left(\mathcal{E}^{\prime}\right)$ are different. From the previous argument, they have the same singular points. Now, $\mathcal{E}$ and $\mathcal{E}^{\prime}$ are in the extension family $\operatorname{Ext}^{1}\left(\mathcal{I}_{Z}(1,1), \mathcal{O}_{Q}\right)$, which is isomorphic to $H^{1}\left(\mathcal{I}_{Z}(-1,-1)\right)^{*}$. From the short exact sequence

$$
0 \rightarrow \mathcal{I}_{Z}(-1,-1) \rightarrow \mathcal{I}_{Z} \rightarrow \mathcal{O}_{C_{H}} \rightarrow 0,
$$

where $C_{H}$ is a smooth conic that does not intersect with $Z$, we have

$$
0 \rightarrow H^{1}\left(\mathcal{I}_{Z}\right)^{*} \rightarrow H^{1}\left(\mathcal{I}_{Z}(-1,-1)\right)^{*} \stackrel{\text { res }}{\rightarrow} H^{0}\left(\mathcal{O}_{C_{H}}\right)^{*} \rightarrow 0
$$

Here, the map ' $r e s^{\prime}$ sends to $\mathcal{E}$ to $\left.\mathcal{E}\right|_{C_{H}}$. Note that $H^{1}\left(\mathcal{I}_{Z}\right)^{*}$ is a corank 1-subspace of $H^{1}\left(\mathcal{I}_{Z}(-1,-1)\right)^{*}$.

If we choose $H$ properly so that the image of $H^{1}\left(\mathcal{I}_{Z}\right)^{*}$ contains $\mathcal{E}$, but not $\mathcal{E}^{\prime}$, then their splitting will be different. To be precise, we have

$$
\left.\mathcal{E}\right|_{C_{H}}=\mathcal{O}_{C_{H}}(-2 p) \oplus \mathcal{O}_{C_{H}},\left.\mathcal{E}^{\prime}\right|_{C_{H}}=\mathcal{O}_{C_{H}}(-p)^{\oplus 2},
$$

where $p$ is a point on $C_{H}$. In particular, $S(\mathcal{E})$ and $S\left(\mathcal{E}^{\prime}\right)$ are different.

In fact, the argument in the proof of (3.1) can be applied to any symmetric determinantal cubic hypersurface with 4 singular points, we obtain the following:

Corollary 3.4. $\mathfrak{M}(4)$ is biratonal to the variety of the symmetric determinantal cubic hypersurfaces with 4 singular points whose corresponding hyperplanes have 4 intersection points on $Q$.

Proof. It is known in 2] that cubic surfaces with 4 rational double points are projectively isomorphic to the Cayley 4-nodal cubic surface, which is a cubic surface with 4 nodal points defined by

$$
t_{0} t_{1} t_{2}+t_{0} t_{1} t_{3}+t_{0} t_{2} t_{3}+t_{1} t_{2} t_{3}=\operatorname{det}\left(\begin{array}{ccc}
t_{0} & 0 & t_{2} \\
0 & t_{1} & -t_{2} \\
-t_{3} & t_{3} & t_{2}+t_{3}
\end{array}\right),
$$

which has 4 nodal points $[1,0,0,0],[0,1,0,0],[0,0,1,0],[0,0,0,1]$. It means that we have a 3-dimensional family of cubic symmetroids for each fixed 4 points as singularities. Here $3=\operatorname{dim} \operatorname{PGL}(4)-\operatorname{dim}\left(\mathbb{P}_{3}^{[4]}\right)$. So the assertion follows automatically from the previous theorem because the dimension of the variety of the cubic symmetroids in the assertion is $11=\operatorname{dim}(\mathrm{PGL}(4))-$ 4, which is the dimension of $\mathfrak{M}(4)$.

Theorem 3.5. $\mathfrak{M}(4)$ is rational.

Proof. Let us prove that the variety $Y$ of the cubic symmetroids with 4 singular points whose corresponding hyperplanes have 4 intersection points on $Q$, is rational. First of all, the variety $X$ of cubic symmetroids with 4 singular points, generically has a $\mathbb{P}_{3}$-bundle structure over $\mathbb{P}_{3}^{[4]}$ and it is transitively acted by PGL(4). Thus $X$ is rational and we have a dominant map $\pi: X \rightarrow \mathbb{P}_{3}^{[4]}$ to a rational variety $\mathbb{P}_{3}^{[4]}$. Since $Y$ is a subvariety of $X$ 
that is generically a $\mathbb{P}_{3}$-bundle over $Q^{[4]}$ from $\pi$ and $Q^{[4]}$ is rational, so $Y$ is a rational variety.

Now let us consider a special case when $Z$ is coplanar. In this case, $S(\mathcal{E})$ is a cubic surface with a unique singular point corresponding to the hyperplane containing $Z$, say $H$. Note that $h^{0}(\mathcal{E}(1,1))=2$. Then there are 1-dimensional family of zero-dimensional subscheme $Z$ for which $\mathcal{E}$ fits into the sequence (11). Such $Z$ should be contained in $C_{H}$. For each $Z$, we can consider $\mathbb{P}_{1}$-family of hyperplanes that contain two points of $Z$ and this corresponds to a line contained in $S(\mathcal{E})$. So we can find 6 lines contained in $S(\mathcal{E})$ out of one such $Z$. As we vary $Z$ in the 1 -dimensional family, we have infinitely many lines through $H$ contained in $S(\mathcal{E})$. Thus we obtain the following statement:

Proposition 3.6. For the vector bundle $\mathcal{E}$ fitted into the sequence (1) with coplanar $Z, S(\mathcal{E})$ is a cone over a cubic curve in $\mathbb{P}_{2}^{*}$ with the vertex point corresponding the hyperplane containing $Z$.

\section{REFERENCES}

[1] Fabrizio Catanese, Footnotes to a theorem of I. Reider, Algebraic geometry (L'Aquila, 1988), Lecture Notes in Math., vol. 1417, Springer, Berlin, 1990, pp. 67-74. MR 1040551 (91d:14022)

[2] Igor Dolgachev, Topics in classical algebraic geometry - part 1, Lecture Notes, http://www.math.lsa.umich.edu/ idolga/topics1.pdf.

[3] D. Gieseker, On the moduli of vector bundles on an algebraic surface, Ann. of Math. (2) 106 (1977), no. 1, 45-60. MR 466475 (81h:14014)

[4] Robin Hartshorne, Algebraic geometry, Springer-Verlag, New York, 1977, Graduate Texts in Mathematics, No. 52. MR 0463157 (57 \#3116)

[5] Sukmoon Huh, Jumping conics on a smooth quadric in $\mathbb{P}_{3}$, Ann. Mat. Pura Appl. (4) 190 (2011), no. 2, 195-208. MR 2786169 (2012d:14072)

[6] Moduli of stable sheaves on a smooth quadric and a Brill-Noether locus, J. Pure Appl. Algebra 215 (2011), no. 9, 2099-2105. MR 2786600 (2012e:14088)

[7] J. Le Potier, Lectures on vector bundles, Cambridge Studies in Advanced Mathematics, vol. 54, Cambridge University Press, Cambridge, 1997, Translated by A. Maciocia. MR 1428426 (98a:14019)

[8] Hiraku Nakajima, Lectures on Hilbert schemes of points on surfaces, University Lecture Series, vol. 18, American Mathematical Society, Providence, RI, 1999. MR 1711344 (2001b:14007)

Department of Mathematics, Sungkyunkwan University, Cheoncheon-dong, JANGAN-GU, SUWON 440-746, KoreA

E-mail address: sukmoonh@skku.edu 